Overall,15\% (44/300) were registered in PROSPERO. This proportion has increased steadily over time and was 29\% (15/ $52)$ in 2018. Therapeutic SRs were more frequently registered than SRs with another focus, with 18\% (22/123) and 12\% (22/177), respectively. We did not observe a clear trend regarding the country.

Median number of authors and time from submission to publication did not differ between registered and non-registered SRs. Median time from search to submission was slightly shorter in registered than in non-registered SRs (14 vs 17 weeks), but median time from search to publication was longer (51 vs 43 weeks).

Of the 44 analyzed PROSPERO records, most (82\%) have been registered before the date of last search. For 23 records $(52 \%)$ only one version existed, which means they have never been updated. Only six records (14\%) were up-to-date, i.e. their status was 'completed and published' and a link to the publication was available.

Conclusions More SRs are being registered in PROSPERO each year, but only few records are up-to-date. PROSPERO should be further promoted among prospective authors of SRs. Current users of PROSPERO need to be reminded to keep their records up-to-date. This could be done through PROSPERO or by journals when authors submit a manuscript for a SR.

\section{PREDATOR PUBLISHING OR FAKE SCIENCE? A CASE SERIES OF 75 UNSOLICITED EMAILS RECEIVED FROM 'PREDATOR JOURNALS.'}

Drew Dagens. Royal Air Force, Centre for Aviation Medicine, UK

\subsection{6/bmjebm-2019-EBMLive.5}

Objectives The rise of 'fake news' has achieved notoriety in the popular press over the last few years. In the scientific press a similar problem, known as 'predator publishing' has arisen. There is currently great debate about the nature and extent of 'predator publishing.' An informal consensus would suggest that junior academics are inundated by requests for articles from journals of dubious quality. These same early career academics are thought to be particularly vulnerable to the pressure to 'publish or be damned.'

Relatively little data is published on the quantitative extent of this problem or its effect on Evidence Based Medicine. Here is described a case series of 75 sequential journal requests received by a postgraduate student over a 4-month period in response to a short letter to Critical Care regarding a fungal assay. The 75 paper requests were analysed for features that might make them be considered 'predatory.'

Method

1) 75 consecutive unsolicited email requests from publications requesting article submission were collected and analysed.

The emails were all received to the personal email account of an English-speaking MSc student over a three-month period.
Most cited this researcher's recent academic publications.

2) The following data was extracted from each email:

- Journal name

- Publisher

- Was the journal fee paying?

- Was the journal registered with PubMed? Medline?

- What article processing charges were there?

- ISSN

- Impact factor (Web of Science)

3) Suggestions that the publication may be a 'predator journal' were sought:

- Was the email overly flattering in tone?

- Was the journal relevant?

- Did the journal permit email submissions?

- Was the journal on 'Beal's list' of predatory publications?

- Was the journal on the BIH 'whitelist' of Open Access Journals?

4) In addition, invitations to speak at conferences/to become an editor were collated

Results 75 emails were analysed. The emails requested submissions on topics as varied as 'archives of animal husbandry and dairy science,' and 'Current Trends in Civil and Structural Engineering.'

The majority (65\%) were from 5-6 publishers who have been described as 'predator publishers' elsewhere.

- All 75 journals were fee-paying

- Only 4 were registered with MEDLINE

- 23 has selected citations on MEDLINE

- 10 could not be found on PUBMED

- The mean average requested APC was $\$ 1690$

- Only 44 journals clearly displayed ISSN numbers.

- 33 of the email requests were phrased in language that could be described as effusive or sycophantic.

- Only 25 bored any relevance to the original article submitted to critical care.

- 50 of the journals accepted email submissions.

- Only 2 of the journals appeared on the BIH 'whitelist'

- 58 appeared on 'Beal's list,' a well-known archive of 'nuisance or 'predator' publications.

- 20 speaking requests, 5 editorial invitations received.

Conclusions This case series describe email requests to publish received by a junior academic over a 4-month period. During this short time the researcher received over 75 requests. The majority were from a small number of publishers, all of which have been accused of producing 'predatory journals' in the past. The vast majority appeared on easily accessed 'blacklists' and only three appeared on commonly used 'white lists.'

The evidence of this case series suggests that junior academics are exposed to 'predator publications.'

However, these papers are so easily identified as suspect that it is hard to imagine anyone being genuinely fooled by them. It would be wiser to acknowledge to remove the passive 'predator' description and acknowledge that these journals are co-produced by the scientific community. 
Perhaps it is time to make a paradigm shift and move from calling from the phrase 'predator journals' to the more appropriate 'fake science.'

\section{UNEXPLAINED DISCREPANCIES BETWEEN PLANNED AND CONDUCTED STATISTICAL ANALYSES IN RANDOMISED TRIALS}

${ }^{1}$ Brennan Kahan, ${ }^{2}$ Suzie Cro, ${ }^{3}$ Gordon Forbes, ${ }^{2}$ Nicholas Andrew Johnson. ${ }^{1}$ Pragmatic Clinical Trials Unit, Queen Mary University of London, London, UKi ${ }^{2}$ Imperial Clinical Trials Unit, Imperial College London, London, UK; ${ }^{3}$ Department of Biostatistics and health informatics, Institute of Psychiatry, Psychology and Neuroscience, Kings College London, London, UK

\subsection{6/bmjebm-2019-EBMLive.6}

Objectives To evaluate how often pre-specified statistical analysis approaches were publicly available for randomised trials, and the frequency of unexplained discrepancies between planned and conducted analyses.

Method We reviewed randomised trials published in six general medical journals (BMJ, JAMA, Lancet, NEJM, PLOS Medicine, and Annals of Internal Medicine) from JanuaryApril 2018. Main outcome measures were (i) the number of trials with a publicly available pre-specified analysis approach for the primary outcome (in a protocol or statistical analysis plan $[\mathrm{SAP}]$ ); (ii) the number of trials with no unexplained discrepancies between the trial publication and the pre-specified approach; and (iii) the types of unexplained discrepancies. Discrepancies were classified as unexplained unless the change was specified in a subsequent version of the protocol or SAP or the discrepancy was discussed in the trial publication. Data extraction was performed independently by two reviewers.

Results Overall, 89 of 101 eligible trials (88\%) had a publicly available pre-specified analysis approach (83 in a protocol, 6 in a SAP); this document was dated after recruitment began for 27/89 trials (30\%), and for 21 trials (24\%) no date was available.

Only 22/89 trials (25\%) did not have any unexplained discrepancies ( $\mathrm{n}=5$ no discrepancies, $\mathrm{n}=17$ explained discrepancies only). Fifty-four trials (61\%) had one or more unexplained discrepancies, and in 13 trials (15\%) it was impossible to ascertain whether any unexplained discrepancies occurred due to incomplete reporting of the statistical methods in the trial publication. Unexplained discrepancies were most common for the analysis model $(n=30,34 \%)$ and analysis population $(n=28,31 \%)$, followed by the use of covariates $(n=23,26 \%)$ and handling of missing data $(n=16,18 \%)$.

Most trials did not report the blinding status of the statistician in relation to database access or final sign-off of the SAP. Conclusions Discrepancies in the statistical analysis approach were common. We identified several barriers preventing an evaluation of whether changes may have introduced bias: (i) many protocols and analysis plans were from after recruitment began, preventing a comparison with the pre-trial analysis approach; (ii) discrepancies were rarely explained or justified in the trial publication; (iii) the blinding status of statisticians in relation to modifications of analysis methods was rarely reported; and (iv) some descriptions of the analysis methods used in the final publication were inadequate, preventing a comparison with the pre-specified approach. Resolution of these barriers is likely to require a multi-faceted approach targeting investigators, journals, and trial registry websites.

\section{PAPERS AS LIVING DOCUMENTS: USING LITERATE PROGRAMMING TO PRODUCE FULLY TRANSPARENT, REPRODUCIBLE RESEARCH MANUSCRIPTS}

1,2Matthew Parkes. 'Arthritis Research UK Centre for Epidemiology, University of Manchester, Manchester, UK: ${ }^{2}$ NIHR Manchester Musculoskeletal Biomedical Research Centre, Central Manchester University Hospitals NHS Foundation Trust, Manchester Academic Health Science Centre, Manchester, UK

\subsection{6/bmjebm-2019-EBMLive.7}

Objectives The existing model of academic writing and publishing in medical research has not strayed far from its correspondence-based letter writing origins. Authors frequently complain that this system is out of date and restrictive. Currently, articles lack transparency - it is difficult to fully and concisely explain complex analyses without presenting full code and dataset structure. Analyses are also fixed and limited - should readers wish to test assumptions, conduct additional tests, or amalgamate study data with other datasets, they are limited to that which is published, or to contacting authors for datasets and code (often unsuccessfully).

Literate programming is an approach which allows manuscripts to be more transparent, reproducible, and interactive. There are free, open-source tools (e.g. RMarkdown and Pweave) which allow entire research manuscripts to be generated end-to-end in one continuous interwoven block, with live code which runs upon opening the document, which can be compiled into .pdf or HTML.

Method This abstract demonstrates how manuscripts can be written using the literate programming model. Using a cleaned, publicly available dataset taken from Vickers' 2006 paper (Vickers Trials 2006, 7:15), we use the knitr, rmarkdown, and rticles packages in RStudio, to write a fully transparent example trial results paper. This paper is compiled into .pdf format using the PLOS LaTeX template (Public Library of Science 2018; https://journals.plos.org/plosone/s/latex), a commonly used manuscript template which is under Creative Commons licence.

Results The resulting . pdf manuscript has a format familiar to readers, yet has increased transparency and interactivity, as the code and dataset are distributed with, and are integrated in, the manuscript. The script can be therefore be downloaded and altered to test assumptions, derive values not presented in the paper (to be used in meta-analyses, for example), and reproduce results.

The compiled paper will be available at the author's GitHub repository: https://github.com/mattyjparkes/EBMLive2019-example-paper

Conclusions These 'living papers', whereby code, data, and interpretation are all interwoven into one live-compiled document has numerous applications for meta-level analyses, and a significantly greater level of transparency. They circumvent the limitations discussed with the current static publishing model, allowing readers to interact and unpick papers in a way that is currently not possible with manuscripts that are divorced from their datasets and analysis code. 